1 Health Justice Initiative, Cape Town, South Africa

2 School of Public Health and Family Medicine, University of Cape Town, South Africa

3 Yale School of Public Health, New Haven, USA

Cite this as: BMJ 2021;375:n3074 http://dx.doi.org/10.1136/bmj.n3074 Published: 13 December 2021

\section{Unequal global vaccine coverage is at the heart of the current covid-19 crisis}

\section{The emergence of the omicron variant shows the risks of vaccine inequity, and the response of the global North has been to further discriminate against and isolate the global South}

\section{Fatima Hassan, ${ }^{1}$ Leslie London, ${ }^{2}$ Gregg Gonsalves ${ }^{3}$}

In early 2020, as the covid-19 pandemic started to spread across the world, warm words of global solidarity claimed that "we are all in this together." But these were little more than platitudes. From vaccine inequity, to blocking the TRIPS waiver, and selective travel bans-the global South has consistently been treated in a paternalistic and racist way. ${ }^{1}$

Before the Omicron variant emerged, the pandemic was regarded as almost "over" in many developed countries with higher rates of covid-19 vaccination and the rollout of booster programmes. Now, with a new variant and ongoing uncertainty about its impact, politicians are scrambling to slam shut borders, often without scientific justification. Selective travel bans are misguided attempts to firefight the symptoms of inequality in global access to vaccine supplies. By now, we all know that no one is safe until everyone is safe.

So let us be clear on how we have ended up in this situation-uneven and slow access to covid-19 vaccines in the global South has brought us to this point. There is a way out of this, but pharmaceutical companies are intent on not sharing knowledge to help Africa manufacture vaccines and speed up production, despite taking huge injections of public funding. This is not only unethical, but inequitable and unsustainable. Unequal access to vaccines risks creating a breeding ground for variants.

Recently, a narrative emerged that vaccine "supply" cannot be a barrier to vaccine rollouts, because in late 2021, governments in the global South deferred supplies as uptake slowed slightly. ${ }^{23}$ It is worth noting that other countries have over ordered vaccine doses and then wasted them when they have not been used. The argument that supply is not an issue, is premised on vaccine hesitancy alone as the cause of slowed uptake, but it does not factor in the lack of access to timely supplies of vaccine doses as highlighted by the WHO, and the impact of worrying anti-vaccine campaigns everywhere. ${ }^{4-6}$ It provides convenient cover to pharmaceutical companies whose control of markets have fuelled artificial scarcity. The fact is only $6.3 \%$ of people in LICs received one dose with only about $7 \%$ vaccinated in Africa. ${ }^{78}$

Substantially more doses have been administered to people in high income and upper middle income countries. ${ }^{9}$ As well as the risk of new variants emerging in unvaccinated populations, this lack of vaccines in the global South means that the health and socio-economic burden of covid-19 cases, hospital admissions, and deaths will fall disproportionately on these countries.

There is evidence that many people in Africa want vaccination, but are not getting it in a timely manner. Recent studies show that there is a greater willingness among people in the global South than in some parts of the global North. ${ }^{1011}$ Moreover, the mere existence of hesitancy in several parts of the global North, has never been invoked as a reason not to prioritise supplies to them.

One study published in Nature analysed vaccine acceptance across 10 LMICs in Asia, Africa, and South America, compared to Russia and the US. ${ }^{11}$ It found a higher willingness to have a vaccine in LMICs compared with the US and Russia "primarily explained by an interest in personal protection against covid-19, while concern about side effects is the most common reason for hesitancy.” And it concluded that "prioritising vaccine distribution to the global South should yield high returns in advancing global immunisation coverage.” This is what we should collectively and expeditiously work towards.

Significantly, most Northern countries started their vaccination rollouts in the first quarter of 2021 while LMICs are still waiting for sufficient, timely supplies relying on a drip feed system, which requires rationing of the rollout programme. ${ }^{5}$ The result: 1 in 4 in African healthcare workers are vaccinated compared to at least $75 \%$ of healthcare workers in the global North. ${ }^{12}$

South Africa, like many other African countries, had limited supplies for much of the past year. Throughout the first three quarters of 2021, the national vaccine programme was beset by material delays, because companies entered into pre-agreements with preferred "customers" and with limited manufacturing partners. ${ }^{13} 14$

Contract negotiations also took several months due to manufacturers' demands: state procurement, unfettered export and allocation rights, non-disclosure, price determination, and broad indemnification against liability. ${ }^{15}$ The non-transparency of vaccine contracts, underpinned by excessive Intellectual Property protection, exacerbates suspicion, even of those who procure vaccines.

As new data on variants emerged, vaccine selection changed, often requiring urgent programmatic adjustments at a national level. Throughout this time, pharmaceutical companies have had the power to 
decide almost everything, including the control of production and distribution that made coherent planning and effective implementation impossible. Vaccines produced in South Africa were exported to Europe in mid-2021 while South Africa faced a third wave of covid-19, while vaccine doses with short expiry dates were "donated" creating a strain on fragile health systems..$^{15-17}$ This created a confusing stop-start strategy, due to production bottlenecks and erratic distribution, all of which remain non-transparent.

And here, South Africa is no exception, it's challenges are illustrative of a wider malaise in this pandemic characterised by secrecy, racism, and a lack of transparency.

Competing interests: none declared

Provenance and peer review: commissioned, not peer reviewed

1 World trade organization. Waiver from certain provisions of the TRIPS agreement for the prevention, containment, and treatment of covid-19 https://docs.wto.org/dol2fe/Pages/SS/directdoc.aspx?filename=q:/IP/C/W669R1.pdf\&Open=True

2 Brown $\mathrm{G}$. The Independent. Boris Johnson has a dangerous disregard for the facts over efforts to vaccinate the world. https://www.independent.co.uk/voices/covid-omicron-vaccine-borisjohnson-b1965600.html

3 South Africa delays COVID vaccine deliveries as inoculations slow. November 2021. https://www.reuters.com/world/africa/exclusive-south-africa-delays-covid-vaccine-deliveries-inoculations-slow-2021-11-24/

4 Johnson T, Moultrie T, Gonsalves G, Hassan F. An inconvenient truth: The real reason why Africa is not getting vaccinated. https://bhekisisa.org/opinion/2021-10-12-an-inconvenient-truth-thereal-reason-why-africa-is-not-getting-vaccinated/

5 Health Justice Initiative. HJI Summary Sheets (Vaccine Supplies). https://healthjusticeinitiative.org.za/2021/10/13/hji-summary-sheets-vaccine-supplies/

6 WHO. WHO Director-General's opening remarks at the media briefing on COVID-19-8 December 2021. https://www.who.int/director-general/speeches/detail/who-director-general-s-openingremarks-at-the-media-briefing-on-covid-19-8-december-2021

7 Our World in Data. Coronavirus (COVID-19) Vaccinations. https://ourworldindata.org/covid-vaccinations

8 Africa CDC. Coronavirus Disease 2019. https://africacdc.org/covid-19/

9 Our World in Data. Covid-19 vaccine doses administered per 100 people, by income group. https://ourworldindata.org/grapher/cumulative-covid-vaccinations-income-group?country=High+income Low+income $\sim$ Lower+middle+income Upper+middle+income

10 Prevent epidemics. https://preventepidemics.org/covid19/perc/

11 Solís Arce JS, Warren SS, Meriggi NF, etal. COVID-19 vaccine acceptance and hesitancy in lowand middle-income countries. Nat Med 2021;27:1385-94. doi: 10.1038/s41591-021-01454-y. pmid: 34272499

12 WHO. Only 1 in 4 African health workers fully vaccinated against COVID-19. https://www.afro.who.int/news/only-1-4-african-health-workers-fully-vaccinated-against-covid19

13 Health Justice Initiative. Timeline of key South Africa COVID-19 related developments and Health Justice Initiative's engagement with government on vaccine access for South Africa. https://healthjusticeinitiative.org.za/vaccine-equity/vaccine-timeline/

14 Human Rights Watch. "Whoever Finds the Vaccine Must Share It." https://www.hrw.org/sites/default/files/media_2020/11/globalvaccine1020_insert_LOWRES_WITH_COVER.pdf

15 The New York Times. Covid Vaccines Produced in Africa Are Being Exported to Europe. https://www.nytimes.com/2021/08/16/business/johnson-johnson-vaccine-africa-exported-europe.html

16 Malan M. No teachers will get J\&J jabs this week - the US first has to rule that the shots are safe. https://bhekisisa.org/article/2021-06-06-no-teachers-will-get-jj-jabs-this-week-the-us-firsthas-to-rule-that-the-shots-are-safe/

17 BBC News. Malawi burns thousands of expired AstraZeneca Covid-19 vaccine doses. https://www.bbc.co.uk/news/world-africa-57168841 